\title{
Children's multiplicative transformations of discrete and continuous quantities
}

\section{Citation}

Barth, Hilary, Andrew Baron, Elizabeth Spelke, and Susan Carey. 2009. "Children's Multiplicative Transformations of Discrete and Continuous Quantities." Journal of Experimental Child Psychology 103 (4) (August): 441-454. doi:10.1016/j.jecp.2009.01.014.

\section{Published Version}

10.1016/j.jecp.2009.01.014

\section{Permanent link}

http://nrs.harvard.edu/urn-3:HUL.InstRepos:33010404

\section{Terms of Use}

This article was downloaded from Harvard University's DASH repository, and is made available under the terms and conditions applicable to Open Access Policy Articles, as set forth at http:// nrs.harvard.edu/urn-3:HUL.InstRepos:dash.current.terms-of-use\#OAP

\section{Share Your Story}

The Harvard community has made this article openly available.

Please share how this access benefits you. Submit a story.

Accessibility 
RUNNING HEAD: Multiplicative transformations

Keywords: Ratio sensitivity, ratios, multiplicative operations, doubling, halving

Children's multiplicative transformations of discrete and continuous quantities Hilary Barth ${ }^{1}$, Andrew Baron ${ }^{2}$, Elizabeth Spelke ${ }^{2}$, and Susan Carey ${ }^{2}$

\footnotetext{
${ }^{1}$ Wesleyan University

${ }^{2}$ Harvard University
}

Word count: 6733 words

Corresponding author: Hilary Barth (hbarth@wesleyan.edu), Department of Psychology, Wesleyan University, 207 High St., Middletown, CT 06459.

Author note: The authors thank Lacey Beckmann for assistance with data collection. This work was supported by NSF grant REC-0087721 to S. Carey and E. Spelke, by a National Academy of Education/Spencer Foundation Postdoctoral Fellowship to H. Barth, and by a Wesleyan University Project Grant to H. Barth. Address correspondence to Hilary Barth (hbarth@wesleyan.edu) at the Department of Psychology, Wesleyan University, 207 High St, Middletown CT 06459. 


\section{Abstract}

Recent studies have documented an evolutionarily primitive, early emerging cognitive system for the mental representation of numerical quantity (the analog magnitude system). Studies with non-human primates, human infants, and preschoolers have shown this system to support computations of numerical ordering, addition, and subtraction involving whole number concepts prior to arithmetic training. Here we report evidence that this system supports children's predictions about the outcomes of halving and perhaps also doubling transformations. A total of one hundred and thirty-eight kindergarten and first grade children were asked to reason about the quantity resulting from the doubling or halving of an initial numerosity (of a set of dots) or an initial length (of a bar). Controls for dot size, total dot area, and dot density ensured that children were responding to the number of dots in the arrays. Prior to formal instruction in symbolic multiplication, division, or rational number, halving (and perhaps doubling) computations appear to be deployed over discrete and possibly continuous quantities. The ability to apply simple multiplicative transformations to analog magnitude representations of quantity may form a part of the toolkit that children use to construct later concepts of rational number. 


\section{Children's multiplicative transformations of discrete and continuous quantities}

The ability to represent approximate numerical magnitudes without the use of language is common to humans of all ages and to nonhuman animals. Animals, infants, children, and adults prevented from applying their exact verbal counting skills discriminate sets based on their cardinal values (for animals and human adults, see Dehaene, 1997, for a review; for human infants and children, see for example, Xu and Spelke, 2000; Barth, La Mont, Lipton, \& Spelke, 2005; Cordes and Brannon, 2008). An "analog magnitude representation" system appears to underlie this ability: the discrete numerosity of the set is internally coded by a mental magnitude, with the magnitude proportional to the number of elements in the set. Like comparative judgments of many kinds of continuous quantities, comparative judgments of discrete number are least accurate when the ratio of compared numerosities is closest to 1:1. Ratio-dependent discrimination in accord with Weber's Law is a key signature of the analog magnitude system (Gallistel \& Gelman, 1992, 2000; Dehaene, 2007).

Analog magnitude representations support computations of numerical ordering, addition, and subtraction across species and throughout the course of development. Most relevant here is evidence that nonverbal animals as well as human infants and children use analog magnitude representations to compute the outcomes of additive operations over visually presented sets of elements (McCrink \& Wynn, 2004; Barth et al., 2005; Flombaum, Junge, \& Hauser, 2005; Slaughter, Kamppi, \& Paynter, 2006; Cantlon \& Brannon, 2007; Gilmore, McCarthy, \& Spelke, 2007; Barth, Beckmann, \& Spelke, 2008).

More controversial is the question of whether analog magnitude representations of approximate number also support multiplicative operations on sets before young children receive formal training in multiplication and division. Concepts of multiplicative, rather than additive, 
change are critical to children's later construction of an understanding of rational number (Smith, Solomon, \& Carey, 2005), a famously difficult achievement of middle school math. It is often suggested that children's early intuitions about quantity transformations may support later learning about fractions, but these intuitions are often thought to rest on protoquantitative, nonnumerical notions of amount (Confrey, 1994; Mix, Levine, \& Huttenlocher, 1999; Resnick \& Singer, 1993; Resnick, 1992). To our knowledge, the potential role of analog magnitude representations of discrete quantity in children's intuitive knowledge of multiplicative transformations has not yet been investigated.

Gelman and Gallistel (Gallistel \& Gelman, 1992, 2000, 2005) hold that mental magnitudes representing number (like those representing non-numerical quantity) do enter into ordering, addition, subtraction, multiplication, and division operations, even in the brains of nonverbal animals (Gallistel, 1990; Leon \& Gallistel, 1998; Gallistel, Mark, King, \& Latham, 2001; but see Church \& Broadbent, 1990; Kakade \& Dayan, 2002; Yang \& Shadlen, 2007 for alternative views). On this view, analog magnitudes provide a common representational format permitting computations over both continuous and discrete quantities.

Some human adult studies also appear consistent with this idea: adults succeed at tasks which may involve multiplying and dividing approximate numerical magnitudes, even when they are prevented from exact counting (Barth, 2002). Because adults have had many years of arithmetic instruction, however, they may have solved these tasks by forming verbal estimates of the quantities involved and then invoking symbolic multiplication or division. Studies of patients with calculation deficits support this latter possibility, because impairments in symbolic multiplication have been linked to impairments in language but not in nonsymbolic number 
processing (Cohen, Dehaene, Chochon, Lehéricy, \& Naccache, 2000; Lemer, Dehaene, Spelke, \& Cohen, 2003).

Representations of both discrete and continuous quantity do appear to support simpler forms of reasoning about multiplicative relationships. Adults track proportions unconsciously and make use of them when transferring from a discrimination learned for a continuous quantity to a novel discrimination of discrete quantity (Balci \& Gallistel, 2006). A recent study has shown that even infants spontaneously represent the ratios between two sets of dots, discriminating new arrays with the same ratios of blue to red dots as those they have seen before from arrays in which the dots are in a different ratio relationship (McCrink \& Wynn, 2007). In young children, much previous work on proportional reasoning has focused on continuous quantity. Though some studies have reported earlier competence in proportional reasoning about continuous vs. discrete quantity, these often involve discrete tasks that provide opportunities for exact counting. Children's apparent lack of competence could stem not from difficulties in reasoning about discrete quantities per se, but from the tendency to count when a task affords the opportunity (Jeong, Levine, \& Huttenlocher, 2007; Boyer, Levine, \& Huttenlocher, 2008).

Here we focus on a simple form of multiplicative reasoning: the ability to apply the multiplicative transformations of halving or doubling to continuous or discrete quantities. We ask children to observe a few examples of such transformations, identify the ratio relationship that holds between the original quantity and the transformed one, and then apply the same transformation to a novel quantity, and then judge whether the transformed quantity would be larger or smaller than a comparison quantity. This task requires more than the detection of a ratio relationship: to succeed, children must apply a transformation that operates on an initial quantity to yield a second quantity that is a fixed ratio of the first. 
Some evidence suggests that young children may not succeed at these tasks. Children exhibit an understanding of additive relations between quantities before they develop an understanding of multiplicative relations (Resnick \& Singer, 1993), and numerous studies from the tradition of information integration theory suggest that younger children apply additive integration rules rather than normative multiplicative rules (e.g. Schlottmann \& Anderson, 1994; Wilkening \& Anderson, 1991; Wilkening, 1982; Anderson \& Cuneo, 1978; but see Gigerenzer $\&$ Richter, 1990). These results have led researchers to argue that multiplicative reasoning is not available at all to children under the age of seven or eight.

In contrast, other studies have found evidence of intuitive reasoning about multiplicative transformations in younger children, provided that the task situation only required the modification of a single quantity (Schlottman \& Tring, 2005; Schlottman, 2001). Also, Confrey and her colleagues have argued that young children possess schemas that form the basis for reasoning about multiplicative operations without relying on repeated addition, proposing that children show intuitive insight into a conceptual primitive called "splitting" that supports later reasoning about ratio, proportion, multiplication, and division (e.g. Confrey, 1994).

The present studies address the following questions. First, is there evidence that analog magnitude representations of number can support computations of halving or doubling in young children? We tested kindergarten and first grade children, who have no formal instruction in symbolic multiplication or division, or in symbolic representations of fractions. We used a task in which stimuli are presented rapidly, to discourage attempts at exact counting, and in which a single numerical quantity is transformed, in order to maximize children's chances of success. Second, are multiplicative computations evident earlier, or more robustly, for continuous 
quantities than for discrete quantities? To address this question, we adapted the same procedure to a task in which the child mentally doubled or halved the magnitude of a continuous quantity.

\section{Experiment 1: Continuous and discrete doubling}

Kindergarten and first grade children observed a small number of examples of doubling transformations applied to either discrete quantities (blue dot arrays' numerosities) or continuous quantities (blue bars' lengths). Animated sequences presented on computer screens showed an initial quantity that was then covered by an occluder; while the initial quantity was hidden, children heard a sound indicating that a transformation was taking place (a rapid series of tones increasing in pitch). Then the occluder was removed and the resulting quantity (double the magnitude of the initial quantity) was revealed. During test trials, children saw novel quantities that were then occluded, followed by the sound that had previously accompanied the doubling transformation. Children compared the resulting (never presented) quantity to a final quantity (an array of red dots or a red bar).

\section{Method}

\section{Participants}

Thirty first grade children and thirty-four kindergarten children recruited from Massachusetts schools participated in the spring of their school year. Stimuli were presented in the forms of an animated computer game on a Macintosh iBook laptop computer with a screen resolution of 1024 by 768 pixels. Fourteen first graders (mean age 7 years 1 month) and seventeen kindergartners (mean age 6 years 3 months) participated in the Continuous condition, and sixteen first graders (mean age 6 years 11 months) and seventeen kindergartners (mean age 6 years 2 months) participated in the Discrete condition (the length of the testing session precluded 
a within-subjects design). Participants completed a comparison task first to acclimate them to the procedure; the doubling task followed the comparison task.

Procedure

For all tasks in Experiments 1 and 2: Children observed the outcomes of four transformations during the example trials, and received corrective feedback following their guesses during the four practice trials. During test trials, children never saw the outcomes of the transformations; they only heard a sound indicating the transformation's occurrence. Children judged which magnitude was greater while only the red magnitude was visible. Stimulus presentations were brief so that children could not count dots or measure lengths, and red and blue bars were presented in different positions and orientations. Blue dots were always 10 pixels in diameter and red dots were always 3 pixels in diameter. Bars varied only in length, not width, so that children would attend to transformations of length rather than assessing area (Spence, 2004). Ratios of the compared bars' lengths (or sets' numerosities) could have one of three values: $4: 7$ (5 trials), 4:6 (6 trials), or 4:5 (5 trials), with the red bar longer (or the red set larger) on half the trials.

Continuous Comparison task. Children first completed four practice trials to introduce the elements of the task. In the first two practice trials, a blue rectangular bar appeared in the top half of the computer screen, followed by a red rectangular bar in the central region of the bottom part of the screen. The blue bar was rotated up to 45 degrees in either direction from the horizontal, and the red bar was always horizontally oriented. Children were asked to judge which bar was longer. In the second pair of practice trials, the blue bar appeared and was covered after 2.5 seconds with a large occluding rectangle, filling the top portion of the screen. The red bar appeared and the child judged which one was longer (with only the red bar visible during the 
choice). Children received meaningful feedback during the four practice trials. Sixteen comparison test trials (Figure 1A) followed the procedure of the final practice trials except that children received only mildly positive feedback. Lengths ranged from 60 to 240 pixel-widths.

Continuous Doubling task. Children were presented with four example trials first in which there was no task. They saw a blue bar appear on the top half of the screen, which was then covered by a rectangular occluder after 2.5 seconds as in the comparison task. A sound was heard (a rapid sequence of notes, rising in pitch) while the blue bar remained hidden, and then the occluder disappeared to reveal a transformed blue bar twice the length of the original. Four practice trials followed: the blue bar appeared and was covered by the occluder, the "transforming" sound was heard, and a red bar appeared at the bottom of the screen. The child was asked which was longer, the new blue bar or the red bar. In the final step, the occluder was removed to reveal the transformed blue bar; this allowed the child to check their judgment concerning the relative lengths of the transformed blue bar and the red bar. Sixteen test trials followed (Figure 1B): in the test trials, the transformed blue bar was never revealed and children were given mildly positive feedback regardless of response. Children judged which bar was longer while only the red bar was visible. The final comparisons (between the lengths of the never-presented transformed blue bar and the red bar) were matched to the comparisons made in the Continuous Comparison task, so the initial blue bars in the Continuous Doubling task were necessarily half the lengths of those presented during the comparison task. Lengths ranged from 30 to 240 pixels.

Discrete Comparison task. This task followed the procedure of the Continuous Comparison task with sets of dots rather than bars. Blue dots appeared in an invisible rectangular envelope ( 512 by 192 pixels) in the top half of the screen, and red dots appeared in a square 
region outlined in red in the central lower region of the screen (192 by 192 pixel-widths). Sets' numerosities ranged from 12 dots to 80 dots. Test trials are depicted in Figure 2A.

Discrete Doubling task. This task followed the procedure of the Continuous Doubling task with sets of dots rather than bars. Sets of blue dots appeared on the top half of the screen in an invisible rectangular envelope (256 by 192 pixel-widths), which was then covered by a rectangular occluder. When the occluder disappeared to reveal a transformed blue set with twice the number of dots as the initial set (on initial example trials only), the doubled set appeared in a 512 by 192 pixel invisible rectangular envelope, so that density remained constant before and after the transformation while area varied. Red dots were always presented within the same small square region, clearly delineated in red, such that the area covered by the red array stayed roughly constant with changes in red set numerosity, though red set density did vary with numerosity. The final comparisons (between the numerosities of the never-presented transformed blue set and the red set) were matched to the comparisons made in the Discrete Comparison task, so the initial blue sets in the Discrete Doubling task necessarily contained half as many dots as those presented during the comparison task. Set sizes ranged from 6 dots to 80 dots. Test trials are depicted in Figure 2B.

\section{Results}

An ANOVA examined the effects of the between-subjects factors Age and Condition (continuous or discrete) and the within-subjects factors Operation (comparison or doubling) and Ratio $(4: 7,4: 6$, or $4: 5)$ on the percentage correct in the test trials (i.e., which was larger: the hidden blue quantity or the visible comparison red quantity). There was no main effect for age, and age did not interact with any other variable. Accuracy scores for the continuous and discrete comparison and doubling tasks, collapsed across both age groups, are shown in Figure 3. 
Children performed above chance for both operations, in both conditions, at every ratio $(\mathrm{p}<$ 0.05). For the 3 ratios in the continuous comparison task, children were $90 \%, 91 \%$, and $81 \%$ correct with SDs 19\%, 17\%, and 16\%; for continuous doubling, $82 \%, 75 \%$, and $65 \%$ correct with SDs $21 \%, 15 \%, 22 \%$; for discrete comparison, $82 \%, 77 \%$, and $60 \%$ correct with SDs $17 \%$, $18 \%, 16 \%$; for discrete doubling, $70 \%, 72 \%$, and $65 \%$ correct with SDs $15 \%, 16 \%, 16 \%$.

There was a main effect of Operation $(\mathrm{F}(1,60)=19.85, \mathrm{p}<.0005)$ : accuracy was greater for comparison than for doubling. There was a main effect of Ratio $(F(2,120)=27.51, p<.0005)$ with a significant linear trend $(\mathrm{F}(1,60)=42.94, \mathrm{p}<.0005)$. There was also a main effect of betweensubjects factor Condition $(\mathrm{F}(1,60)=20.24, \mathrm{p}<.0005)$; accuracy was greater in the continuous than in the discrete condition. These main effects must be interpreted light of the interaction of Operation and Condition $(\mathrm{F}(1,60)=6.47, \mathrm{p}<.05)$. That is, the main effects were due to children's high accuracy for the continuous comparison task: the discrete comparison and doubling tasks did not differ from each other $(\mathrm{t}(32)=1.57, \mathrm{p}>.05)$, and the continuous and discrete doubling tasks did not differ from each other $(\mathrm{t}(62)=1.93, \mathrm{p}>.05)$. Of course, it is not surprising that accuracy was highest for the continuous comparison task; only in this task were the red and blue stimuli being compared identical except for length (the dimension being compared) and orientation. The dot arrays in the discrete comparison task differed dramatically in overall size, shape, and dot dimensions. Finally, there was a significant three-way interaction of Condition, Operation, and Ratio $(\mathrm{F}(2,120)=5.07, \mathrm{p}<.01)$ due to relatively low accuracy levels on the 4:7 ratio trials in the discrete doubling task.

\section{Discussion}

Children successfully chose which stimulus (the red or the mentally transformed blue) was larger on the doubling tasks in both the continuous and discrete conditions, and the two age 
groups did not perform differently. Just as in previous studies of additive operations subserved by analog magnitude number representations, performance was as accurate on the doubling task as on a comparison task. Performance was above chance for every task at every ratio tested, and accuracy was dependent on the ratio of the quantities being compared, consistent with the signature of the analog magnitude system. Children's overall accuracy was equally high for the doubling tasks in the discrete condition (the transformation of an initial set's numerosity) and in the continuous condition (the transformation of an initial bar's length).

These data are consistent with the children's abstracting the common ratio relation between the two quantities during the practice trials, and then transforming the initial quantity during each test trial according to that ratio (approximately twice as large). However, the demonstrated transformation was equivalent to adding another instance of the initial quantity (“ADD ANOTHER"). Such an additive strategy may be especially likely in the case of length: our finding that the continuous comparison of lengths was by far the easiest task suggests that children can easily create a working memory representation of the length of the blue bar and compare it to the length of a red bar in a different position and orientation. Children's performance patterns in a wide variety of quantitative tasks suggest that they tend to apply additive rules (such as repeated addition), rather than multiplicative rules, until relatively late in elementary school (Resnick, 1992; Ginsburg, 1977). This tendency can occur even when additive rules lead to incorrect results, but in the present study, both additive and multiplicative interpretations were consistent with the transformations that children observed.

If children are only able to make use of additive transformations of analog magnitude representations prior to instruction on symbolic multiplication and division, they might succeed at the doubling task of Experiment 1 but fail at a similar task that does not lend itself to additive 
strategies. To test this hypothesis, children were tested on an analogous halving task in

Experiment 2. Though repeated addition is one way to produce the effect of doubling, there is no comparable additive operation for halving (because subtracting one half requires identifying the half to be subtracted).

\section{Experiment 2: Continuous and discrete halving}

In the second experiment, kindergarten and first grade children completed versions of the tasks of Experiment 1 in which halving transformations replaced doubling transformations.

\section{Method}

Participants.

Twenty-seven first grade children and forty-seven kindergartners recruited from Massachusetts schools participated in the spring of their school year. There were two betweensubjects conditions: fourteen first graders (mean age 7 years 1 month) and twenty-two kindergartners (mean age 6 years 2 months) participated in the Continuous condition and thirteen first graders (mean age 6 years 11 months) and twenty-five kindergartners (mean age 6 years 1 month) participated in the Discrete condition. As in Experiment 1, stimuli were presented in the form of a computer game, and all participants completed the comparison task first, followed by the halving task.

\section{Procedure}

Continuous condition. The continuous comparison procedure of Experiment 2 was identical to the continuous comparison procedure of Experiment 1 ( Figure 1A). The continuous halving task procedure (Figure 1C) was just like the continuous doubling task procedure (four demonstration trials, four practice trials, and sixteen test trials), except that the sound indicating that a hidden transformation was taking place was now a series of notes of falling pitch (rather 
than rising pitch), and the transformed blue bar was half its original length. Initial blue bar lengths in the Continuous Halving task were matched to those presented in the Continuous Comparison task. Bars' lengths ranged from 30 to 240 pixel-widths.

Discrete condition. The discrete comparison task (Figure 2A) was identical to that described in Experiment 1. The discrete halving task procedure (Figure 2C) was identical to the discrete doubling task procedure except that the sound was now a series of notes of falling pitch (rather than rising pitch), and the transformed blue set was half its original numerosity. The presented initial blue sets in the Discrete Halving task contained as many dots as the blue sets presented during the comparison task. Set sizes ranged from 6 dots to 80 dots.

\section{Results}

An ANOVA examined the effects of between-subjects factors Age and Condition (continuous or discrete) and within-subjects factors Operation (comparison or halving) and Ratio (4:7, 4:6, or 4:5) on accuracy on the test trials. As in Experiment 1, there were no effects of age. Accuracy scores for the continuous and discrete comparison and halving tasks, collapsed across the two age groups, are shown in Figure 4. Children performed above chance for both operations, in both conditions, at every ratio $(\mathrm{p}<0.05)$. For the 3 ratios in the continuous comparison task, children were $97 \%, 95 \%$, and $89 \%$ correct with SDs $11 \%, 11 \%$, and $15 \%$; for continuous halving, $75 \%, 58 \%$, and $62 \%$ correct with SDs $22 \%, 20 \%, 17 \%$; for discrete comparison, $81 \%$, $75 \%$, and $68 \%$ correct with SDs 16\%, 19\%, 19\%; for discrete halving, $69 \%, 65 \%$, and 58\% correct with SDs $21 \%, 18 \%, 23 \%$.

There was a main effect of Operation $(\mathrm{F}(1,70)=109.86, \mathrm{p}<.0001)$ : accuracy was greater for comparison than for halving. There was a main effect of Ratio $(F(2,140)=16.25, \mathrm{p}<.0005)$ with a significant linear trend $(F(1,70)=30.46, \mathrm{p}<.0005)$. There was also an effect of the between- 
subjects factor Condition $(\mathrm{F}(1,70)=17.34, \mathrm{p}<.0005)$ : accuracy was greater in the continuous than in the discrete condition. A significant interaction of Operation and Condition $(F(1,70)=20.62$, $\mathrm{p}<.0005)$ suggested that the main effects were due in part to children's high accuracy for the continuous comparison task, which required comparing the lengths of identical stimuli that differed only in color, orientation and position. All other comparisons required transforming the blue stimuli held in working memory (halving them) and, in the case of the discrete condition, comparing the set sizes of arrays that differed dramatically (in dot size, array size and shape). Accuracy levels on the discrete comparison and halving tasks differed as well $(\mathrm{t}(37)=4.03$, $\mathrm{p}<0.005$ (corrected for multiple comparisons). Accuracy levels on the continuous and discrete halving tasks did not differ from each other $(\mathrm{t}(72)=0.35, \mathrm{p}>.05$, uncorrected $)$. There was a significant three-way interaction of Condition, Operation, and Ratio $(F(2,140)=3.41, \mathrm{p}<.05)$ due to relatively low accuracy levels on the $4: 6$ ratio trials in the continuous halving task.

\section{Discussion}

Children chose the larger quantity with better-than-chance accuracy for the comparison tasks and the halving tasks in both the continuous and discrete conditions at every ratio tested. Accuracy decreased as the ratio of the compared quantities approached 1, consistent with the signature of the analog magnitude system. Kindergarten and first grade children did not perform differently. These results - ratio sensitivity, no age effect, and no difference between continuous and discrete halving - are remarkably convergent with the doubling results of Experiment 1 . But before we discuss the implications of children's success at doubling and halving both discrete and continuous quantities, we must explore other strategies children may have adopted in these tasks. Might children have succeeded without carrying out any computation on the hidden 
quantity? Each alternative we explored makes specific predictions regarding details of the data; we tested for the use of alternative strategies by testing those predictions as follows.

\section{Alternative strategy 1: Ignore the transformation altogether}

Did children simply compare the initially presented blue magnitude to the red magnitude, ignoring the invisible transformation of the blue magnitude? This possibility is especially important to consider because comparison did precede doubling or halving for all participants, in order to accustom children to the elements of the doubling \& halving tasks. Such a strategy would result in chance performance overall on the doubling task if children applied it consistently, because the presented blue quantity (before it was invisibly "doubled") was always smaller than the red quantity. Therefore such a strategy would lead to $100 \%$ (or very high) accuracy on the trials with a correct answer of "red" and $0 \%$ (or very low) accuracy on trials with a correct answer of "blue." Because children performed above chance overall, they did not rely entirely on a strategy involving comparing the initial blue quantity to the red quantity. The data suggest that children did not use this strategy even on a subset of the presented trials in the doubling task: if they had done so, they would have achieved higher levels of accuracy on the trials with a correct answer of "red" than on those with a correct answer of "blue." This is not the case for the continuous doubling task ("answer = red" trials, 74\%; "answer = blue" trials, 74\%) or for the discrete doubling task ("answer = red" trials, 73\%; "answer = blue" trials, 65\%; $\mathrm{t}(32)=1.25, \mathrm{p}>.05)$

This strategy would also result in chance performance if children applied it consistently in the halving task, because the initially presented blue quantity (before its unseen "halving") was always larger than the red quantity. Because children performed above chance overall, they did not rely entirely on this strategy. If children applied the strategy on a subset of the presented 
trials, they would achieve higher levels of accuracy on the trials with a correct answer of "blue" than on those with a correct answer of "red." This was not the case for the discrete halving task ("answer $=$ red" trials, 63\%; "answer = blue" trials, 65\%). Thus, children showed no evidence of comparing the initial blue quantity to the final red quantity in the discrete halving task. However, on the continuous halving task, children were $42 \%$ correct for the "answer $=$ red" trials (not significantly different from chance; $\mathrm{t}(35)=1.65, \mathrm{p}>.05$ ) and $88 \%$ correct for the "answer $=$ blue" trials. These accuracy scores were significantly different from each other $(\mathrm{t}(35)=6.49$, $\mathrm{p}<.0001$ ). Children could not have relied entirely on this strategy in the continuous halving task (because such reliance would have produced lower overall accuracy), but the pattern of performance was consistent with the use of a simple comparison strategy on some trials.

\section{Alternative strategy 2: Respond based on range analysis}

Another strategy exists that could have led to better-than-chance performance even if children did not base their choices on the magnitude of the transformed quantity. It is possible that children simply responded based on the range of values of the red lengths or set numerosities (the magnitude presented last in every trial). The correct response for the largest red items was always "red" (these were bigger than the transformed blue item), and the correct response for the smallest red items was always "blue" (these were smaller than the transformed blue item). Children have been found to exploit such a range analysis in previous acrossmodality approximate subtraction tasks (when subtracting numerosities of sound sequences from numerosities of visual sets), though not in analogous addition tasks (Barth, Beckmann, \& Spelke, 2008). It is especially important to consider this strategy in the present doubling task because the comparison trials always preceded the doubling trials, and the range of red items was the same in the comparison and doubling trials. Thus, children had ample opportunity to learn about the 
range of magnitudes being presented. If the practice trials taught children to give roughly equal numbers of "blue" and "red" answers, and the comparison trial block exposed them to a particular range of red set numerosities or red bar lengths, children might well have made use of this strategy. Four distinct arguments are relevant to our consideration of children's use of this range-based strategy.

First, if children learned about the range of red magnitudes from the initial comparison trials, we should expect the use of this range-based strategy to lead to two specific patterns of performance, because the red numerosities or lengths from the comparison blocks were identical to those for the doubling blocks, but the red numerosities or lengths of the halving blocks were smaller than those for the preceding comparison blocks. If children relied on this strategy, they should be more accurate for doubling than for halving. There was no accuracy difference between the discrete doubling and discrete halving tasks $(\mathrm{t}(69)=1.94, \mathrm{p}>0.05$, uncorrected) but continuous doubling performance was better than continuous halving performance $(\mathrm{t}(65)=2.73$, $\mathrm{p}<0.05$, corrected for multiple comparisons). Thus, this analysis militates against a range based strategy in the discrete tasks, but suggests such a strategy might have played a role in the continuous tasks.

Second, if children did make use of a range based strategy, halving tasks should produce a pattern of bias toward blue responses. This was not the case for the discrete halving task, but for the continuous halving task, children did perform better for the "answer = blue" trials than the "answer $=$ red" trials $(88 \%$ correct vs. $42 \%$ correct, $(\mathrm{t}(35)=6.49, \mathrm{p}<.0001)$. Therefore children's performance patterns for the continuous tasks appear consistent so far with the use of a strategy based on the information about the range of red lengths gathered during the initial comparison block, but there is no evidence of such a strategy in the case of the discrete tasks. 
Third, if children gathered information about the range of red magnitudes from the halving or doubling blocks themselves, rather than from the preceding comparison blocks, they should perform better on the second halves of the blocks than on the first halves. This was not the case for any task. For discrete doubling and discrete halving, there was no difference in performance from the first half of the task to the second half $(\mathrm{t}(32)=0.58, \mathrm{p}>0.05$, and $\mathrm{t}(37)=0.13, \mathrm{p}>0.05$, uncorrected, respectively). For the continuous doubling and halving tasks, performance was better on the first half than on the second $(\mathrm{t}(30)=2.14, \mathrm{p}<0.05$, and $\mathrm{t}(35)=2.42, \mathrm{p}<0.03$, uncorrected, respectively).

Fourth, if children pursued this range-based strategy, then they should perform better on trials containing extreme red magnitudes than on trials containing intermediate red magnitudes, when comparison ratios are equated across the two trial types. Better performance on the trials containing extreme red magnitudes would indicate that children were likely influenced by a strategy based on the magnitude of the final red item, although it does not constitute evidence of children's complete reliance on such a strategy. For both continuous and discrete doubling, accuracy was higher on the trial subsets containing red magnitudes at the extreme ends of the range $(\mathrm{t}(30)=4.0, \mathrm{p}<0.0004$, uncorrected, and $\mathrm{t}(32)=8.28, \mathrm{p}<0.0001$, uncorrected, respectively). In contrast, for both continuous and discrete halving, accuracy was no higher for trial subsets containing extreme red magnitudes $(\mathrm{t}(35)=1.96, \mathrm{p}>0.05$, uncorrected, and $\mathrm{t}(37)=1.57, \mathrm{p}>0.05$, uncorrected). This finding makes sense in light of the fact that in the doubling tasks, red magnitudes were identical in the doubling block and the preceding comparison block, such that range information gathered during the comparison block would remain relevant during the doubling block. It may be that the incorporation of comparison trials designed in this manner obscured evidence of children's ability to respond appropriately to the doubling transformations 
by encouraging them to exploit available range-based information. In contrast, in the halving tasks, red magnitudes shifted dramatically from the comparison block to the halving block.

Thus, these analyses militate against either alternative strategy in the discrete halving task. The simple strategy of comparing the initial blue quantity to the final red quantity, ignoring the transformation, may have played some role in children's performance on the continuous halving task, but not on either of the doubling tasks. Furthermore, the analyses provide some evidence that children could have made use of range based alternative strategies in the doubling tasks (especially continuous doubling), but there was no hint of reliance on these strategies for the halving tasks.

\section{General Discussion}

We sought to answer two questions. First, can analog magnitude representations support reasoning about multiplicative transformations of discrete quantities in young children, prior to formal instruction in relevant symbolic algorithms? Second, are multiplicative computations evident earlier, or more robustly, for continuous quantities than for discrete quantities?

Our findings suggest that children's analog magnitude representations of discrete numerical quantity can indeed enter into halving, and perhaps doubling, operations. Though success at a doubling task like that of Experiment 1 could be explained in terms of a tendency to represent the transformation in terms of addition ("initial quantity + initial quantity" rather than “initial quantity doubled"), children's unambiguous success at the discrete halving task of Experiment 2 cannot be explained in an analogous manner (because subtracting one half requires first halving the initial quantity). These findings are therefore consistent with the idea that children can represent an approximate halving operation, and can apply this operation to representations of discrete numerosity, before they are taught symbolic multiplication, division, 
or fraction notation. Children apparently succeeded at recognizing the nature of the halving transformation based on a small number of examples, applying that transformation to novel discrete sets in order to halve them mentally, and comparing the resulting (never-presented) numerosity to a third set. Overall, the results of Experiment 2 provide evidence of nonverbal approximate halving of discrete, and most probably also continuous, quantities.

Children's accuracy depended on the ratio of the compared quantities, consistent with the idea that children made use of analog magnitude representations of quantity in performing the tasks. Followup analyses testing for the use of alternative strategies showed that these data provide clear evidence of children's ability to apply halving transformations to sets of discrete elements. The evidence is somewhat less clear for the continuous quantities tested here (lengths). Thus, we find no evidence that children are more sensitive to multiplicative transformations of continuous magnitude: Children performed equally well for both types of tasks, and patterns of performance for the discrete tasks constitute stronger evidence for children's ability to reason about multiplicative transformations than do patterns of performance for the continuous tasks. Whether younger children might reveal better be able to double or halve continuous than discrete quantities is a topic for further research.

\section{What multiplicative computations might children have been carrying out?}

It is important to acknowledge that these data provide no evidence that children can multiply or divide one analog magnitude (e.g., approximately 15) by another (e.g., approximately 2). Although we have designated the transformations "doubling" and "halving," we do not mean to imply that representations of the number two enter into the computation. We have no evidence concerning exactly what computation the child is carrying out; after all, it is possible they are multiplying and dividing by two. But it is also possible that they are computing ratios and then 
creating a representation of a new quantity that is a constant ratio to each standard. Whichever computation they are using, especially in the halving task, it is a multiplicative one.

\section{Potential limitations of these studies}

This approach to investigating children's intuitions about multiplicative transformations of quantity required the use of a between-subjects design, due to the length of the test session, the number of conditions tested, and the young age of the participants. Because groups were formed by semi-random assignment rather than being equated with respect to sex, intelligence, attentional resources, or other variables, it is possible that between-group variations may have influenced the results. In future studies it would be ideal to employ within-subjects tests of both continuous and discrete quantities, and within-subjects tests involving halving and doubling transformations. In addition, we chose to design the tasks such that some aspects of the stimuli were balanced across doubling and halving conditions (the overall stimulus magnitudes employed, for example); this necessarily meant that other aspects could not be equated (such as the initially-presented magnitudes; see Figures 1 and 2 and Procedures). Ideally, future studies would explore possible stimulus magnitude effects that may have resulted from this design.

\section{Potential relation to the later construction of rational number concepts}

Both articulated and intuitive concepts of division play an important role in children's eventual construction of an understanding of rational number. An articulated model of fractions based on division is strongly related to middle school children's understanding of other aspects of rational number (Smith, Solomon, \& Carey, 2005), and many researchers suggest that early intuitions about transformations of continuous physical amounts support later fraction learning (Resnick \& Singer, 1993; Confrey, 1994; Moss \& Case, 1999). But children's great difficulty in understanding fractions emphasizes the conceptual distance between intuitions about physical 
quantities and formal reasoning about rational numbers. There may be no clear path from reasoning about amounts in the world to reasoning about numbers as mathematical entities, and we do not yet possess a full description of the intermediate steps children take along this path. Many researchers have argued that learning about fractions requires conceptual change (Smith et al., 2005; Gelman, 1991; Gelman \& Meck, 1992) rather than the enrichment of existing knowledge about numbers and quantities (Mix et al., 1999; Sophian, Geryantes, \& Chang, 1997). On the former view, making sense of rational number is especially difficult for children because their early concept of numbers - that numbers are what you get when you count - must be changed fundamentally when they are confronted with fractions (Smith et al., 2005; Gelman \& Meck, 1992; Hartnett \& Gelman, 1998). To understand rational number, children must come to form a new concept of number as infinitely divisible.

Physical continuous quantities have often been supposed to provide a convenient model for children's thinking about repeated division, because it seems plausible that continuous models of these quantities might be perceptually given. However, a recent clinical interview study demonstrated that this aspect of children's thinking about physical quantity may itself be painstakingly constructed. Despite the apparent perceptual availability of the continuity of matter and length, the study found that many children between eight and twelve years of age do not yet possess a continuous model of matter or (in a pilot study) length (Smith et al., 2005). Such an understanding may be crucial to the construction of an understanding of rational number: all children who showed evidence of a discontinuous model of matter also did not yet understand that numbers could be divided infinitely, and all children who understood the infinite divisibility of number also showed evidence of a continuous model of matter (Smith et al., 2005). 
Early intuitions about transformations of continuous physical amounts, therefore, may not provide children with the conceptual tools they need to build rational number concepts. A concept of repeated division differentiated from subtraction, in the context of reasoning about numbers and physical quantities in verbal tasks, appears to be an important component of older children's construction of concepts of rational number (Smith et al., 2005), yet an understanding of differentiated division in such contexts is relatively late-emerging. The present study shows that even much younger children already appear to possess the ability to apply simple multiplicative transformations to numerical quantities (sets of discrete elements). It is possible that the early nonverbal understanding of halving computations operating over analog magnitude representations of discrete quantity may serve as one of the building blocks of the later understandings of division that are crucial to children's understanding of fractions.

We did not find evidence that intuitive reasoning about multiplicative transformations of continuous quantities preceded such reasoning in the context of discrete quantity, though in the verbally-based clinical interview study described above, children's patterns of response suggested that an understanding of the infinite divisibility of matter appeared to precede that of number (though the acquisition of concepts of the repeated division of numbers and the repeated division of matter progressed largely in parallel, Smith et al., 2005). It may be that early concepts of multiplicative change develop in the context of continuous quantities first (Mix, Huttenlocher, \& Levine, 2002). It is also possible that the developmental patterns observed in studies of young children's nonverbal reasoning about sets and amounts will not parallel those observed in studies of older children's verbal reasoning about numbers and matter. Additional studies are needed to distinguish between these possibilities, and to determine whether the capabilities demonstrated by children in these experiments extend to other simple forms of multiplicative transformations 
beyond halving and doubling (or even to true computations of multiplication and division). Future work will explore the ways in which children's later steps on the path toward rational number concepts might build upon their early ability to perform computations of halving and doubling over analog magnitude representations of discrete quantity. 


\section{References}

Anderson, N., \& Cuneo, D. O. (1978). The height + width rule in children's judgments of quantity. Journal of Experimental Psychology: General, 107, 335-378.

Balci, F., \& Gallistel, C. R. (2006). Cross-domain transfer of quantitative discriminations: Is it all a matter of proportion? Psychonomic Bulletin and Review, 13, 636-642.

Barth, H., Beckmann, L., \& Spelke, E. (2008). Nonsymbolic, approximate arithmetic in children: Evidence for abstract addition prior to instruction. Developmental Psychology, 44, 14661477.

Barth, H., La Mont, K., Lipton, J., and Spelke, E. (2005). Abstract number and arithmetic in preschool children. Proceedings of the National Academy of Sciences, 102, 14116-14121.

Barth, H. (2002). Numerical cognition in adults: Representation and manipulation of nonsymbolic quantities. Unpublished doctoral dissertation, MIT.

Boyer, T., Levine, S., \& Huttenlocher, J. (2008). Development of proportional reasoning: Where young children go wrong. Developmental Psychology, 44, 1478-1490.

Cantlon, J., \& Brannon, E. (2007). Basic math in monkeys and college students. PloS Biology, 5, 2912-2919.

Church, R. M., \& Broadbent, H. A. (1990). Alternative representations of time, number, and rate. Cognition, 37, 55-81.

Cohen, L., Dehaene, S., Chochon, F., Lehéricy, S., \& Naccache, L. (2000). Language and calculation within the parietal lobe: a combined cognitive, anatomical, and fMRI study. Neuropsychologia, 38, 1426-1440.

Confrey, J. (1994). Splitting, similarity, and the rate of change: New approaches to multiplication and exponential functions. In G. Harel \& J. Confrey (Eds.), The development of 
multiplicative reasoning in the learning of mathematics (p. 293-332). Albany, NY: SUNY Press.

Cordes, S., \& Brannon, E. (2008). The difficulties of representing continuous extent in infancy: using number is just easier. Child Development, 79, 476-489.

Dehaene, S. (2007). The number sense. New York: Oxford University Press.

Flombaum, Junge, \& Hauser, M. (2005). Rhesus monkeys (Macaca mulatta) spontaneously compute addition operations over large numbers. Cognition, 97, 315-325.

Gallistel, C, R. (1990). The organization of learning. Cambridge, MA: MIT Press.

Gallistel, C. R., \& Gelman, R. (1992). Preverbal and verbal counting and computation. Cognition, 44, 43-74.

Gallistel, C. R., \& Gelman, R. (2000). Non-verbal numerical cognition: from reals to integers. Trends in Cognitive Sciences, 4, 59-65.

Gallistel, C. R., Mark, T., King, A., \& Latham, P. (2001). The rat approximates an ideal detector of changes in rates of reward: Implications for the law of effect. Journal of Experimental Psychology: Animal Behavior Processes, 27, 354-372.

Gallistel, C. R., \& Gelman, R. (2005). Mathematical cognition. In K. Holyoak \& R. Morrison (Eds.), The Cambridge handbook of thinking and reasoning. Cambridge: Cambridge University Press.

Gelman, R. (1991). Epigenetic foundations of knowledge structures: Initial and transcendent constructions. In S. Carey and R. Gelman, (Eds.). The epigenesis of mind: Essays on biology and cognition. Hillsdale, NJ: Erlbaum Associates. 
Gelman, R., \& Meck, B. (1992). Early principles aid initial but not later conceptions of number. In J. Bideaud, C. Meljac, \& J-P. Fischer (Eds.), Pathways to number: Children's developing numerical abilities. Hillsdale, NJ: Lawrence Erlbaum.

Gigerenzer, G., \& Richter, H. (1990). Context effects and their interaction with development: Area judgments. Cognitive Development, 5, 235-264.

Ginsburg, H. (1977). Children's arithmetic: The learning process. New York: Van Nostrand. Hartnett, P., \& Gelman, R. (1998). Early understandings of number: Paths or barriers to the construction of new understandings? Learning and Instruction, 8, 341-374.

Jeong, Y., Levine, S., \& Huttenlocher, J. (2007). The development of proportional reasoning: Continuous versus discrete quantities. Journal of Cognition and Development, 8, 237-256.

Kakade, S., \& Dayan, P. (2002). Acquisition and extinction in autoshaping. Psychological Review, 109, 533-544.

Leon, M., \& Gallistel, C. R. (1998). Self-stimulating rats combine subjective reward magnitude and subjective reward rate multiplicatively. Journal of Experimental Psychology: Animal Behavior Processes, 24, 265-277.

Lemer, C., Dehaene, S., Spelke, E., \& Cohen, L. (2003). Approximate quantities and exact number words: dissociable systems. Neuropsychologia, 41, 1942-1958.

McCrink, K., \& Wynn, K. (2004). Large-number addition and subtraction by human infants. Psychological Science, 15, 776-781.

McCrink, K., \& Wynn, K. (2007). Ratio abstraction by 6-month-old infants. Psychological Science, $18,740-745$.

Mix, K., Levine, S., \& Huttenlocher, J. (1999). Early fraction calculation ability. Developmental Psychology, 35, 164-174. 
Mix, K., Huttenlocher, J., \& Levine, S., (2002). Quantitative development in infancy and early childhood. New York: Oxford University Press.

Moss, J., \& Case, R. (1999). Developing children's understanding of rational numbers: A new model and an experimental curriculum. Journal for Research in Mathematics and Education $30,122-147$.

Resnick, L. (1992). From protoquantities to operators: Building mathematical competence on a foundation of everyday knowledge. In G. Leinhardt, R. Putnum, \& R. Hattrup (Eds.), Analyses of arithmetic for mathematics teachers. Erlbaum: Hillsdale, NJ.

Resnick, L., \& Singer, J. (1993). Protoquantitative origins of ratio reasoning. In T. Carpenter, E. Fennema, T. and Romberg, T. (Eds.), Rational numbers: An integration of research. Erlbaum: Hillsdale, NJ.

Schlottmann, A. (2001). Children's probability intuitions: Understanding the expected value of complex gambles. Child Development, 72, 103-122.

Schlottmann, A., \& Tring, J. (2005). How children reason about gains and losses: Framing effects in judgement and choice. Swiss Journal of Psychology, 64, 153-171.

Schlottmann, A., \& Anderson, N. (1994). Children's judgments of expected value. Developmental Psychology, 30, 56-66.

Slaughter, V., Kamppi, D., \& Paynter, J. (2006). Toddler subtraction with large sets: further evidence for an analog-magnitude representation of number. Developmental Science, 9, 3339.

Smith, C., Solomon, G., \& Carey, S. (2005). Never getting to zero: Elementary school students' understanding of the infinite divisibility of number and matter. Cognitive Psychology, 51, $101-140$. 
Sophian, C., Garyantes, D., \& Chang, C. (1997). When three is less than two: Early developments in children's understanding of fractional quantities. Developmental Psychology, 33, 731-744.

Spence, I. (2004). The apparent and effective dimensionality of representations of objects. Human Factors, 46, 738-747.

Wilkening, F., \& Anderson, N. (1991). Representation and diagnosis of knowledge structures in developmental psychology. In Anderson, N. (Ed.) Contributions to information integration theory, volume III: Developmental. New Jersey: Erlbaum.

Wilkening, F. (1982). Children's knowledge about time, distance, and velocity interrelations. In W. Friedman (Ed.), The Developmental Psychology of Time. New York: Academic press, pp. $87-112$.

Xu, F., \& Spelke, E. (2000). Large number discrimination by human infants. Cognition 74, B1B11.

Yang, T., \& Shadlen, M. (2007). Probabilistic reasoning by neurons. Nature, 447, 1075-1080. 


\section{Figure Captions}

Figure 1. Schematic depictions of procedures for the continuous comparison task (Experiments 1 and 2), continuous doubling task (Experiment 1), and continuous halving task (Experiment 2). Not drawn to scale.

Figure 2. Schematic depictions of procedures for the discrete comparison task (Experiments 1 and 2), discrete doubling task (Experiment 1), and discrete halving task (Experiment 2). Not drawn to scale.

Figure 3. Children's accuracy for the continuous and discrete comparison and doubling tasks of Experiment 1 (collapsed across kindergarten and first grade participants). Error bars represent SEM.

Figure 4. Children's accuracy for the continuous and discrete comparison and halving tasks of Experiment 2 (collapsed across kindergarten and first grade participants). Error bars represent SEM. 
Figure 1

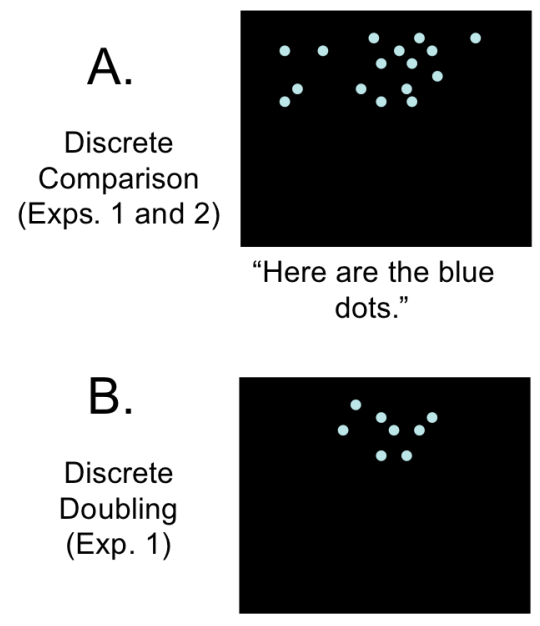

"Here are the blue dots."

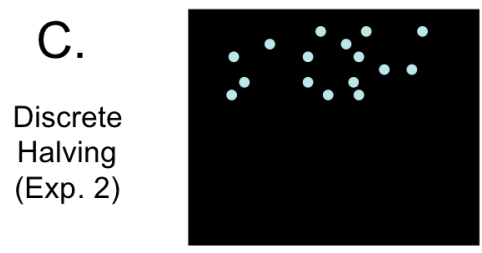

"Here are the blue dots."

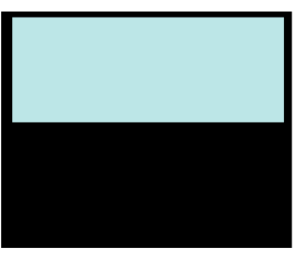

"Now they're behind this box."

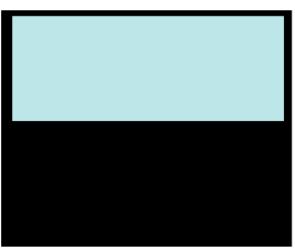

"Now they're behind this box."

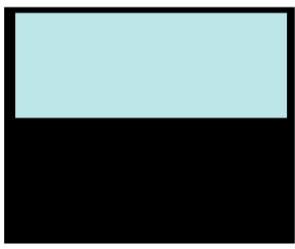

"Now they're behind this box."

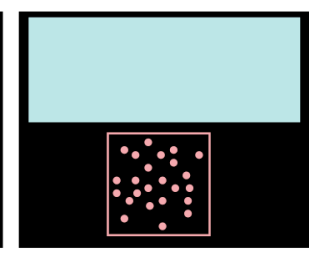

"Here are the red dots. Are there more blue dots or more red dots?'
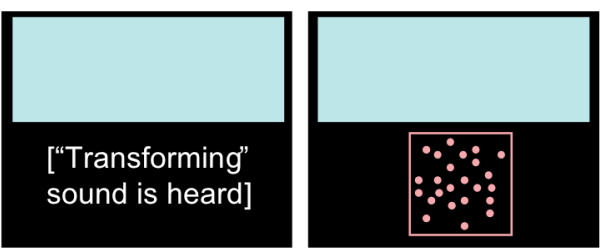

"Now the sound "And here are the red dots. magically changes Now are there more blue them!" dots or more red dots?'

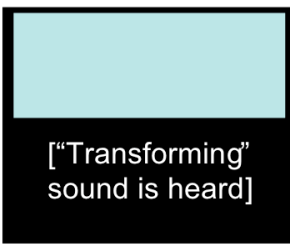

"Now the sound "And here are the red dots. magically changes them!" Now are there more blue dots or more red dots?'

Figure 2

A.

Continuous

Comparison

(Exps. 1 and 2)

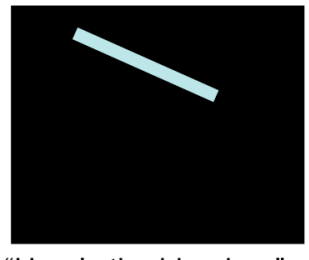

"Here's the blue bar."

B.

Continuous Doubling (Exp. 1)

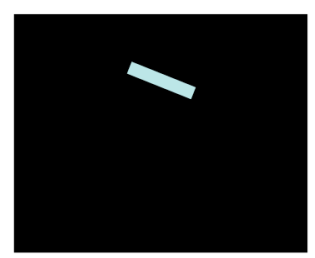

"Here's the blue bar."

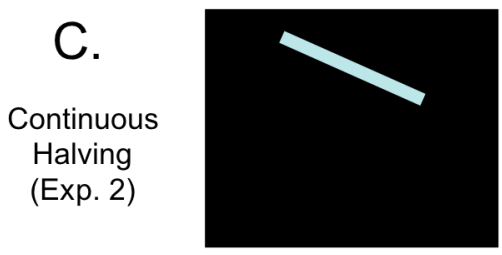

"Here's the blue bar."

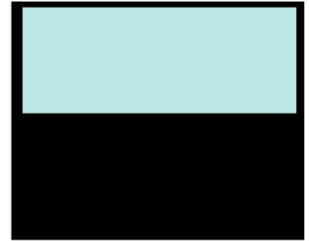

"Now it's behind this box."

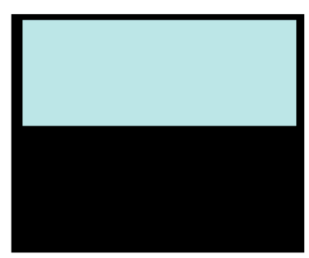

"Now it's behind this box."

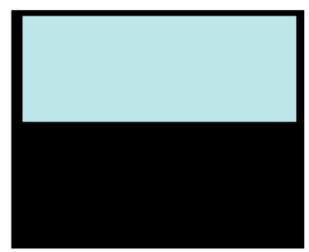

"Now it's behind this box."

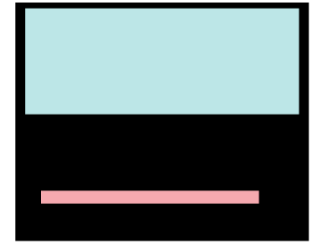

"Here's the red bar. Is the blue bar longer or is the red bar longer?"

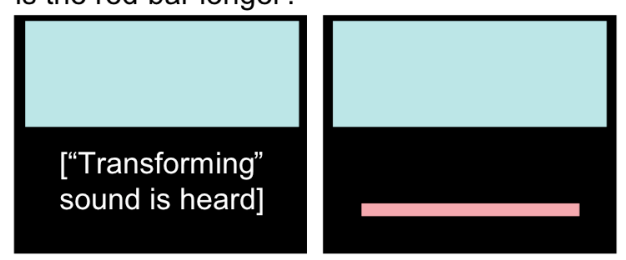

"Now the sound "And here's the red bar. magically changes it!" Is the blue bar longer or is the red bar longer?"
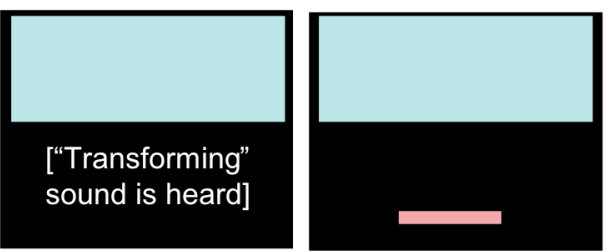

"Now the sound "And here's the red bar. magically changes it!" Is the blue bar longer or is the red bar longer?" 
Figure 3

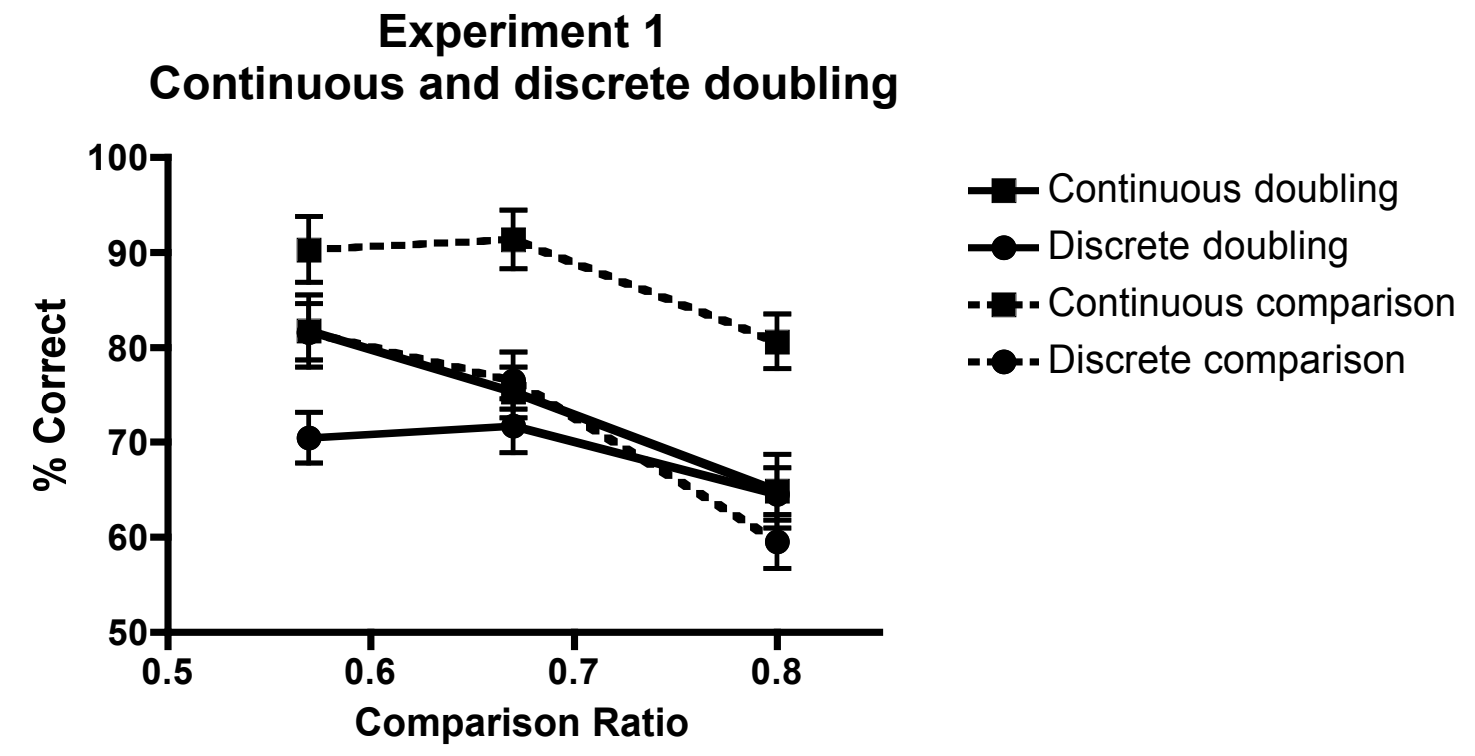

Figure 4
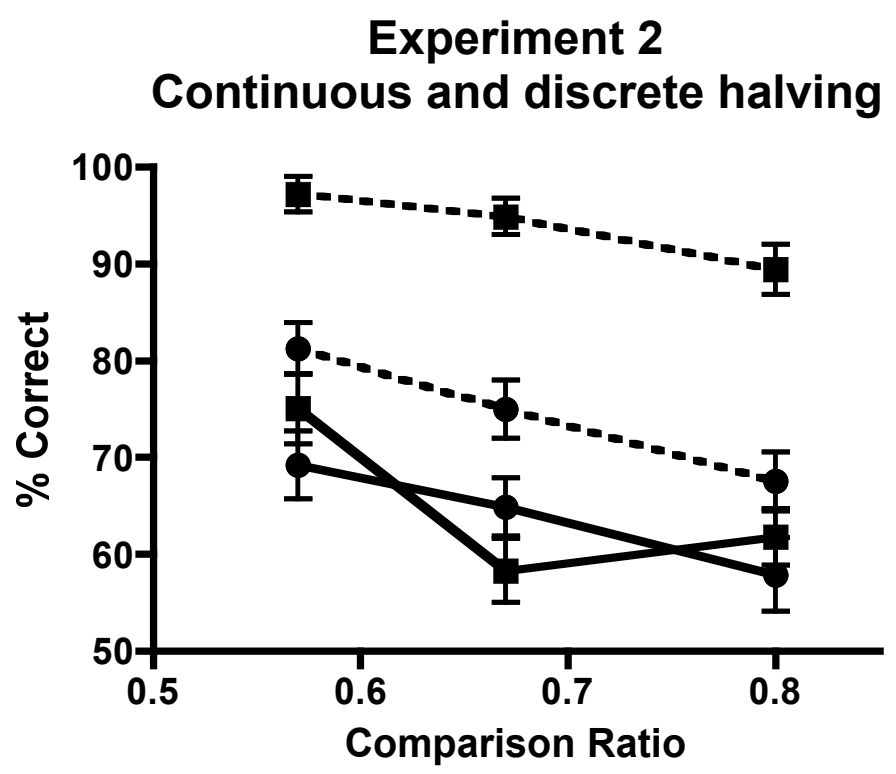

- Continuous halving

- Discrete halving

- - - Continuous comparison

- - Discrete comparison 